Research Article

\title{
Optimal Design of Diversion Piers of Lateral Intake Pumping Station Based on Orthogonal Test
}

\author{
Jiren Zhou, ${ }^{1}$ Miaomiao Zhao, ${ }^{1}$ Chuan Wang $\triangle,{ }^{1}$ and Zhenjun Gao ${ }^{2}$ \\ ${ }^{1}$ College of Hydraulic Science and Engineering, Yangzhou University, Yangzhou 225009, China \\ ${ }^{2}$ College of Mechanical and Power Engineering, China Three Gorges University, Yichang 443002, China \\ Correspondence should be addressed to Chuan Wang; wangchuan198710@126.com
}

Received 16 December 2020; Revised 11 March 2021; Accepted 18 March 2021; Published 26 March 2021

Academic Editor: Yong Zhu

Copyright (c) 2021 Jiren Zhou et al. This is an open access article distributed under the Creative Commons Attribution License, which permits unrestricted use, distribution, and reproduction in any medium, provided the original work is properly cited.

To explore the rectification parameters of the diversion piers optimized for the forebay of the pump station with a lateral angle of $45^{\circ}$, the orthogonal experiment and computational fluid dynamics methods are used to analyze the flow characteristics of the diversion piers under different parameter combinations. The flow pattern in the forebay of the side water inlet is improved. The rectification effect of the diversion piers under 16 schemes is analyzed, considering the length, width, radian, and relative height of the diversion piers. Combined with numerical simulation, a better rectification scheme is provided, and finally, a reasonable range of values for the rectification parameters of the forebay diversion pier of the side $45^{\circ}$ bend angle pump station is obtained.

\section{Introduction}

The forebay of the pumping station is an important water diversion structure, and its hydraulic characteristics directly affect the performance of the pump. The forebay can be divided into frontal water intake and lateral water intake [1-4]. Some pumping stations can only adopt the form of lateral water intake because the layout of pumping stations is often restricted by objective factors, such as water flow characteristics and geographic location. If the water flow from the lateral inflow is oblique to the axis of the inlet pool, then backflow or vortex easily occurs. A number of studies have been carried out to analyze the flow pattern in the forebay and pump. At present, scholars mainly carried out a combination of model test and numerical simulation [5-8]. At the same time, substantial practical engineering problems have been solved using mathematical methods [9-12]. With the development of computer technology, the accuracy of numerical simulation is gradually enhanced and has become the main research method [13-16]. Sweeney [17] used narrow water distribution holes and splitter plate rectification measures to improve the bad flow pattern of the forebay of the lateral diversion pump station and obtained a stable forebay inlet flow pattern. The water inlet conditions of the pump are satisfied, and the hydraulic characteristics are significantly improved. Spence [18] used numerical simulation and model tests to optimize a series of parameters to explore the possible adverse effects of centrifugal pump operation. Various rectification measures are adopted to improve the flow pattern, which is beneficial to better manage the operation of the pump station. Zhou et al. [19] used the pump station model test to analyze the causes of the bad flow pattern in the forebay and proposed corresponding measures to improve the flow pattern. The first domestic research on the vortex at the inlet of the pump inlet channel was based on the Froude model to simulate the prototype flow pattern by increasing the flow velocity by approximately 0.5 times. The results are relatively satisfactory. Cheng et al. [20] quantitatively analyzed the influence of the position, height, angle, and length of the Y-shaped diversion pier on the flow pattern of the pump station's forebay by using Fluent software and obtained the optimal parameters of the Y-shaped diversion pier. Orthogonal experiment was used to analyze multifactor and multilevel experiments. Compared with the full-factor method, the number of experiments using orthogonal experiment was greatly reduced. Using the 
standardized orthogonal table to design the test plan and carry out the range and variance analysis of the test results, the influence law of each factor on the index can be obtained. Then, the optimized production conditions or process conditions can be obtained. Zhou et al. [21] used the orthogonal test method, selected four-factor three-level orthogonal table, analyzed the orthogonal test data, put forward an optimized design model pump scheme, and obtained the influence law of each geometric parameter on the index. Shi et al. [22-24] used a five-factor four-level orthogonal table to design the test plan for the relevant control dimensions of the pump device. Computational fluid dynamics (CFD) numerical simulation was used to calculate each plan, analyze the influence of each parameter on the hydraulic performance of the flow channel, and then verify it with a model test. Finally, the priority order of each control parameter to the index and the optimal plan were obtained. In the present study, numerical simulation is carried out for the forebay of the pumping station with a $45^{\circ}$ lateral bending angle. The orthogonal test method is used to design the geometric parameters of the diversion pier, and the optimized parameter combination is obtained. Then, the CFX numerical calculation is used for analysis and verification. The improvement law of the parameters of the diversion pier on the flow pattern in the forebay of the lateral inlet is obtained.

\section{Study Area and Method}

2.1. Study Area. A pumping station hub with lateral water intake is considered an example. Three pump units are installed in the pump room; each pump has a design flow of $4.0 \mathrm{~m}^{3} / \mathrm{s}$, and the flow channel inlet size is $2.36 \mathrm{~m}$ (width) $\times$ $1.8 \mathrm{~m}$ (length). The intake level of the pumping station is $1.9 \mathrm{~m}$, the elevation of the bottom of the drainage canal is $0.0 \mathrm{~m}$, and the elevation of the forebay bottom is $-1.0 \mathrm{~m}$. The main functions of the project are flood control and drainage. The left side of the pumping station is a self-draining culvert. When the pumping station is in operation, the self-draining culvert control gate is closed. The total width of the outlet of the forebay is $B$, and the length from the center of the inlet of the forebay to the center of the outlet is $L$. Figure 1 shows the plan layout of the intake building of the pumping station, where the lateral bend angle is $45^{\circ}$.

2.2. Study Method. CFX is used to numerically simulate the model, and the overall calculation domain is divided into unstructured grids. The hydraulic loss is used as the evaluation index. After grid independence verification, when the total grid number is 3.58 million, the hydraulic loss is relatively small. Therefore, the influence of the number of grids on the numerical calculation can be ignored.

The inlet boundary condition adopts the quality inlet, the inlet flow channel outlet is selected as the calculated outlet boundary, the standard atmosphere is set to 1 , and the "steel cover assumption" is selected for the surface of the water body, that is, symmetry surface boundary treatment and nonslip walls.

\section{Orthogonal Optimization Design}

3.1. Selection of Test Factors and Levels. Diversion piers are used to improve the flow pattern of the forebay. The geometric parameters of the diversion piers have a certain influence on the inlet channel and the water inlet conditions. The axial uniformity and weighted average angle of the inlet section of the inlet flow passage are used as the quantitative indicators of the test, and the geometric parameters of the diversion pier greatly affect the indicators, which need to be integrated for orthogonal test design. The results of the orthogonal test are analyzed, the optimal parameter combination of the diversion pier is determined, and then the influence of various geometric parameters of the diversion pier on the hydraulic performance of the forebay is studied. The key to this research is to reasonably select the range of geometric parameters of the diversion pier.

For the forebay with different lateral bends, the length of the diversion piers is mainly selected according to the range of its bad flow patterns. The maximum horizontal width of the diversion pier is $1.0 \mathrm{~m}$ because the distance between $1 \#$ and 2\# inlet channels is $1.0 \mathrm{~m}$. The arc-shaped diversion pier can play a good diversion function, and the range of the diversion pier's radian should be selected through repeated trials. The relative height of the diversion pier is used to study the influence of the height of the diversion pier on the hydraulic characteristics of the flow pattern in the forebay, considering that different water levels may occur in the forebay. The schematic of the geometric parameters of the diversion pier is shown in Figure 2, where $l$ is the length of the diversion pier, $b$ is the width of the diversion pier, $\alpha$ is the radian of the diversion pier, and $h$ is the relative height of the diversion pier. The expression is as follows: $h=H_{1} / \mathrm{H}_{2}$. In the formula, $\mathrm{H}_{1}$ is the actual height of the diversion pier, $m ; \mathrm{H}_{2}$ is the water depth in the forebay, $m$.

3.2. Orthogonal Table. In this study, the parameters of the orthogonal experiment are the length, width, radian, and relative height of the diversion pier. The factors are determined according to the design scope. The length of the diversion pier is based on the length of the swing zone in the forebay in the original design. The level of the test factor is determined according to the possible variation range of each factor parameter in the actual project. When the lateral angle is $45^{\circ}$, the orthogonal design of the geometric parameters of the diversion pier in the forebay selects four factors, and each factor selects four levels. The specific parameters of the factor level are shown in Tables 1 and 2. The factor codes $A, B, C$, and $D$ in the table represent the length $(\mathrm{m})$, width $(\mathrm{m})$, radian $\left(^{\circ}\right)$, and relative height of the diversion pier, respectively.

According to the selected factors and the number of levels, the orthogonal table L16 $\left(4^{5}\right)$ is selected for orthogonal design, and one column is the error column.

3.3. Orthogonal Test Results of the Geometric Parameters of the Diversion Pier. The preliminary research analysis shows the position of the bad flow pattern in the forebay, the reason for 


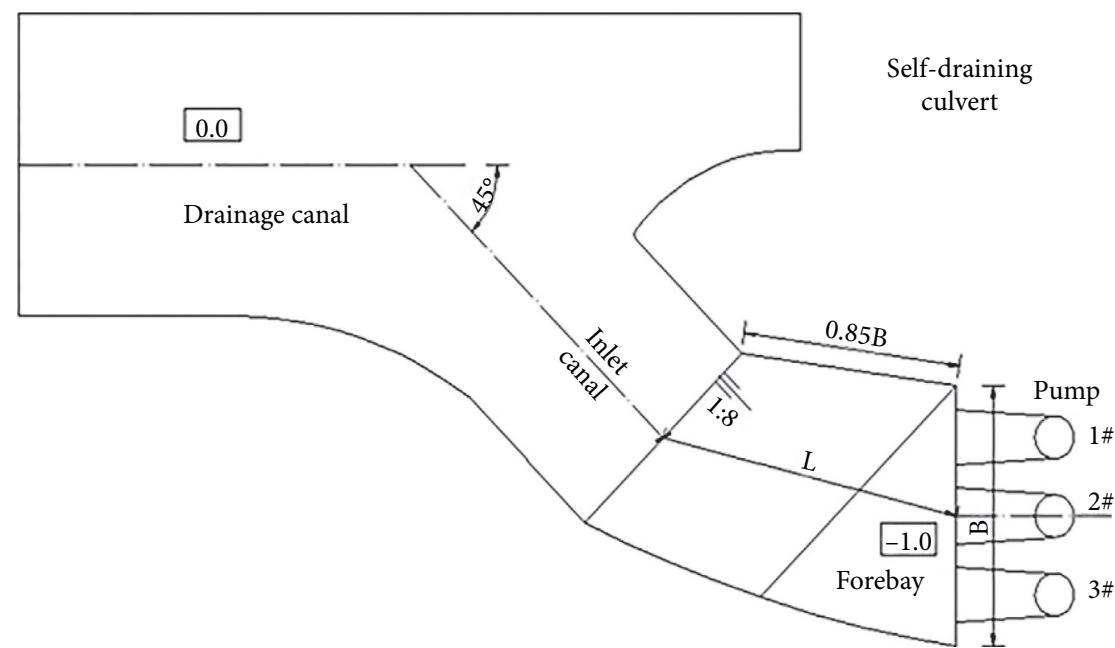

FIGURE 1: Layout of the intake building of the lateral intake pump station.

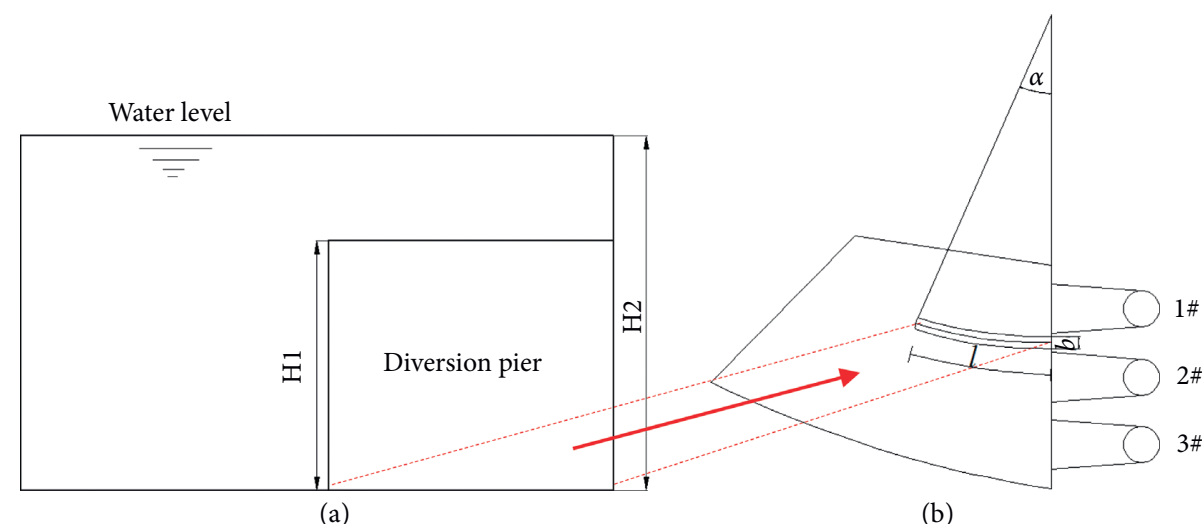

FIGURE 2: Schematic of the geometric parameters of the diversion pier: (a) relative height of diversion pier; (b) diversion pier length, width, and radian.

TABLE 1: Orthogonal table of the forebay of the pumping station with a $45^{\circ}$ lateral angle.

\begin{tabular}{lcccc}
\hline Level & \multicolumn{4}{c}{ Factors } \\
& $\mathrm{A} l / m$ & $\mathrm{~B} b / m$ & $\mathrm{C} \alpha \rho^{\circ}$ & $\mathrm{D} h$ \\
\hline 1 & 7 & 0.4 & 10 & 0.4 \\
2 & 8 & 0.6 & 15 & 0.6 \\
3 & 9 & 0.8 & 20 & 0.8 \\
4 & 10 & 1.0 & 25 & 1.0 \\
\hline
\end{tabular}

its formation, and the approximate range of the recirculation zone when the lateral angle is $45^{\circ}$. The bad flow pattern mainly affects the $1 \#$ inlet flow passage and causes harm to the inlet conditions of the 1 \# inlet flow passage, thereby threatening the normal stability of the pump unit.

Therefore, the engineering measures of adding diversion piers between the 1\# and 2\# inlet channels are considered to improve the flow pattern of the forebay, and the influence of the geometric parameters of the diversion piers on the hydraulic performance of the forebay is studied. The four geometric parameters and levels of the selected diversion piers are designed according to the orthogonal table L16 (45), and each plan is numerically simulated by CFD.
Moreover, the axis of the inlet section of the $1 \#$ inlet channel under each plan is obtained. The uniformity and weighted average angle of flow velocity, test plan, and calculation results are shown in Table 2. Intuitive analysis and variance analysis of the orthogonal test results are carried out, and the influence trend of various factors on the improvement of the inlet conditions of the inlet channel is analyzed. Then, the parameter combination of the optimization of the geometric parameters of the diversion pier is obtained. Finally, the optimized parameter combination obtained is analyzed and verified through numerical calculation to obtain the improvement law of the flow characteristics of the forebay by the geometric parameters of the diversion pier.

3.4. Visual Analysis. Range refers to the difference between the maximum value and the minimum value of the sum of each level index in the same factor, that is, $R=\max \left\{k_{1}, k_{2}, k_{3}, k_{4}\right\}-\min \left\{k_{1}, k_{2}, k_{3}, k_{4}\right\}$ in any column. The value reflects the degree of influence of this factor on the index, that is, the degree of influence of the parameter on the uniformity of the inlet flow velocity of the 1\# inlet flow channel. The larger value indicates greater influence on the 
TABLE 2: Test plan and calculation results.

\begin{tabular}{|c|c|c|c|c|c|c|c|}
\hline Test plan & A & $\mathrm{B}$ & $\mathrm{C}$ & $\mathrm{D}$ & E (error) & Flow rate uniformity (\%) & Weighted average angle $\left({ }^{\circ}\right)$ \\
\hline 1 & 1 & 1 & 1 & 1 & 1 & 84.46 & 70.51 \\
\hline 2 & 1 & 2 & 2 & 2 & 2 & 85.12 & 71.44 \\
\hline 3 & 1 & 3 & 3 & 3 & 3 & 86.77 & 72.65 \\
\hline 4 & 1 & 4 & 4 & 4 & 4 & 90.75 & 72.24 \\
\hline 5 & 2 & 1 & 2 & 3 & 4 & 85.02 & 71.68 \\
\hline 6 & 2 & 2 & 1 & 4 & 3 & 87.23 & 73.28 \\
\hline 7 & 2 & 3 & 4 & 1 & 2 & 84.35 & 70.10 \\
\hline 8 & 2 & 4 & 3 & 2 & 1 & 85.45 & 71.54 \\
\hline 9 & 3 & 1 & 3 & 4 & 2 & 85.16 & 71.93 \\
\hline 10 & 3 & 2 & 4 & 3 & 1 & 86.02 & 71.60 \\
\hline 11 & 3 & 3 & 1 & 2 & 4 & 85.88 & 72.97 \\
\hline 12 & 3 & 4 & 2 & 1 & 3 & 85.28 & 71.23 \\
\hline 13 & 4 & 1 & 4 & 2 & 3 & 84.15 & 70.24 \\
\hline 14 & 4 & 2 & 3 & 1 & 4 & 84.18 & 70.39 \\
\hline 15 & 4 & 3 & 2 & 4 & 1 & 88.06 & 73.70 \\
\hline 16 & 4 & 4 & 1 & 3 & 2 & 87.41 & 74.09 \\
\hline$K_{1}$ & 347.10 & 338.79 & 344.98 & 338.27 & 343.99 & & \\
\hline$K_{2}$ & 342.05 & 342.55 & 343.48 & 340.60 & 342.04 & $T=\Gamma^{16} n-137528$ & \\
\hline$K_{3}$ & 342.34 & 345.06 & 341.56 & 345.22 & 343.43 & $T=\sum_{i=1}^{i} \eta=1375.28$ & \\
\hline$K_{4}$ & 343.80 & 348.89 & 345.27 & 351.20 & 345.83 & & \\
\hline$k_{1}$ & 86.77 & 84.70 & 86.24 & 84.57 & 86.00 & & \\
\hline$k_{2}$ & 85.51 & 85.64 & 85.87 & 85.15 & 85.51 & & \\
\hline$k_{3}$ & 85.58 & 86.26 & 85.39 & 86.31 & 85.86 & $\bar{\eta}=T / 16=85.96$ & \\
\hline$k_{4}$ & 85.95 & 87.22 & 86.32 & 87.80 & 86.46 & & \\
\hline$R_{\mathrm{j}}$ & 1.26 & 2.53 & 0.93 & 3.23 & 0.95 & & \\
\hline
\end{tabular}

uniformity of the flow velocity at the inlet of the inlet flow channel. Table 2 shows that the difference between the parameters and the uniformity of axial flow velocity is $D>B>A>C$. The order of the factors is as follows: the relative height of the diversion pier, the width of the diversion pier, the length of the diversion pier, and the radian of the diversion pier. The research shows that, among the four factors that affect the rectification effect of the diversion pier, the relative height of the diversion pier is the key factor, the length of the diversion pier, the width of the diversion pier are general factors, and the radian of the diversion pier is the secondary factor.

A trend chart is drawn to analyze the specific influence trend of the changes in various factors on improving the uniformity of the flow velocity of the inlet section of the inlet flow channel. The abscissa is the level of each factor, and the ordinate is the average of the sum of the results of the corresponding factors. The relationship among the $k_{i}$ value, the factors, and the uniformity of the flow velocity of the inlet section of the inlet flow channel is shown in Figure 3.

Figure 3 shows that, within the range of the number of factors studied, various factors have different effects on the uniformity of the axial flow velocity of the inlet section of the 1 \# inlet channel. When the length of the diversion pier is $8 \mathrm{~m}$, the index is the smallest, and the index increases below $8 \mathrm{~m}$ and above $8 \mathrm{~m}$. The $20^{\circ}$ radian of the diversion pier leads to the smallest index, and the index is lower than $20^{\circ}$ and higher than $20^{\circ}$. As the width of the diversion pier increases, the index gradually increases, forming a trend that the index increases when the width of the diversion pier continues to increase. When the relative height of the diversion pier changes from 0.4 to 1.0 , the index also increases continuously. This finding indicates that the index reaches the maximum value when the height of the diversion pier is flush with the water surface.

3.5. Analysis of Variance. Variance analysis should be performed on the test results to estimate the size of the error in the test process and analyze whether the influence of various factors on the uniformity of the axial flow velocity at the inlet section of the $1 \#$ inlet flow passage is significant. The orthogonal table leaves an error column to calculate the experimental error. The sum of squared deviations excludes the difference between the factor levels and only represents the size of the experimental error because this column is blank. The results of variance calculation are shown in Table 3. The results show that the variance of the error term is smaller than the variance of each factor. This finding indicates that each factor affects the test index, and no factor is included in the error term. In addition, if the variance and error of the radian of the diversion pier are small, then the change in this factor level has no significant impact on the test results. In addition, the variance and error of other factors are large. Thus, the change in the factor level affects the test results, which have a significant impact. The significant degree of the specific influence of each factor can be judged on the basis of the comparison of the variance ratio and the critical value of $F$.

Table 3 shows that the change in the length of the diversion pier has a certain influence on the index, that is, the uniformity of the axial flow velocity of the inlet section of the 


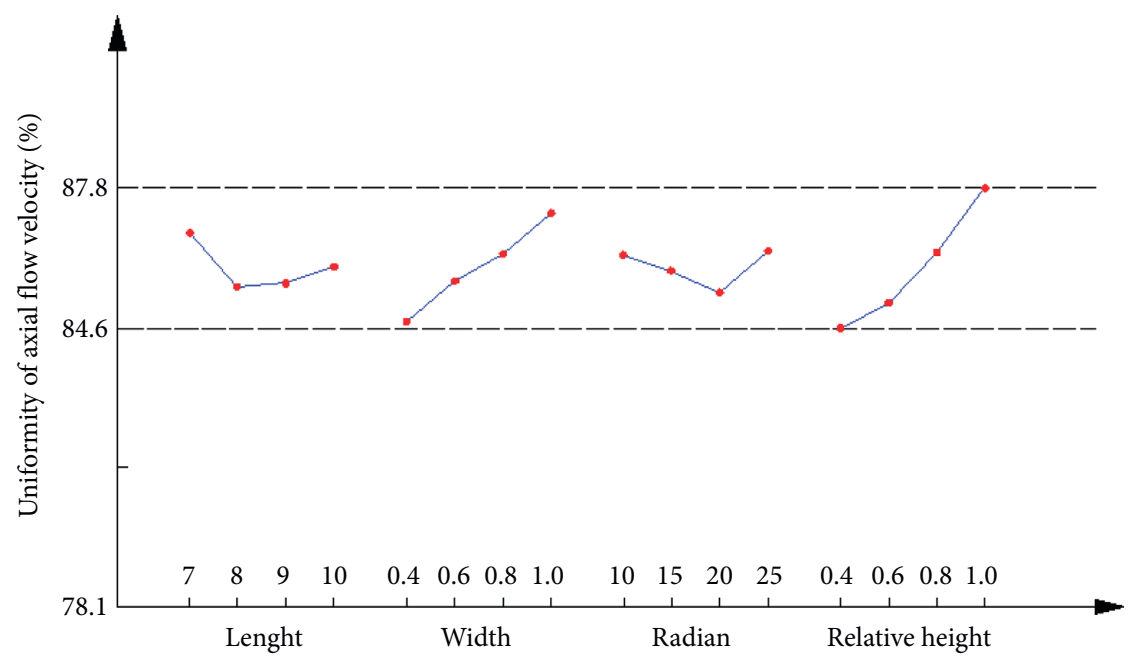

FIGURE 3: Line graph of the relationship between flow velocity uniformity and various factors.

TABLE 3: Analysis of variance of flow velocity uniformity.

\begin{tabular}{lcccccc}
\hline Source of variance & Deviation sum of squares & Degree of freedom & Variance & Variance ratio & $F_{\mathrm{a}}$ & Significant level \\
\hline Length & 3.992 & 3 & 1.331 & 2.016 & $\mathrm{~F}_{0.01}(3,6)=9.78$ & $\mathrm{o}$ \\
Width & 13.508 & 3 & 4.503 & 6.821 & $\mathrm{~F}_{0.05}(3,6)=4.76$ \\
Radian & 2.141 & 3 & 0.714 & & $\mathrm{~F}_{0.1}(3,6)=3.29$ \\
Relative height & 24.375 & 3 & 8.125 & 12.308 & $\mathrm{~F}_{0.25}(3,6)=1.78$ & $* * *$ \\
Error & 1.820 & 3 & 0.607 & & & \\
\hline
\end{tabular}

$1 \#$ inlet channel. The change in the width of the diversion pier significantly affects the index. The change in the radian level of the diversion pier has no significant impact on the index, and the parameters can be arbitrarily selected within the test range according to the specific situation. The change in the relative height of the diversion pier has a very significant impact on the index.

3.6. Determination of Optimal Solution. For the uniformity of axial flow velocity, the larger value is better. General calculation and analysis are performed on the orthogonal test results and the trend graph of the relationship between various factors and indicators, and then, a better test plan is obtained. The maximum average value $k_{i}$ of the axial flow velocity uniformity of the inlet section of the inlet channel of $1 \#$ is selected under the level $i$ of the diversion pier. Figure 3 shows that, among the four levels considered by the length of the diversion pier, the $k_{i}$ value is the largest when the length is $7 \mathrm{~m}$. Among the four levels considered by the width of the diversion pier, the $k_{i}$ value is the largest when the width is $1.0 \mathrm{~m}$. Among the four levels considered by the radian of the diversion pier, the $k_{i}$ value is the largest when the radian is $25^{\circ}$. Among the four levels considered by the relative height of the diversion pier, the $k_{i}$ value is the largest when the relative height is $1.0 \mathrm{~m}$. Therefore, the optimal plan is as follows: the length of the diversion pier is $7 \mathrm{~m}$; the width of the diversion pier is $1.0 \mathrm{~m}$; the radian of the diversion pier is $25^{\circ}$; the relative height of the diversion pier is 1.0. Therefore, the design scheme is plan $A_{1} \mathrm{~B}_{4} \mathrm{C}_{4} D_{4}$.

\section{Analysis of Numerical Simulation Results Based on Orthogonal Experimental Design}

4.1. Feature Section Selection. Two characteristic sections of the horizontal section of the front pond are selected for flow field analysis, namely, two horizontal longitudinal sections of the front pond surface layer and bottom layer. Figure 4 shows the horizontal section, namely, the surface layer a-a and the bottom layer b-b to study the flow pattern and axial velocity distribution of the forebay surface layer and bottom layer, respectively. Among them, the surface layer a-a section is $0.05 \mathrm{~m}$ away from the water surface, and the coordinates of the surface layer and bottom layer are $Z_{a}=1.85 \mathrm{~m}$ and $Z_{b}=0.05 \mathrm{~m}$, respectively.

4.2. Selection of the Flow Pattern Scheme for the Horizontal Profile of the Forebay. The optimal combination of diversion pier parameters is obtained in accordance with the calculation of orthogonal experiment. According to this parameter setting, several schemes are numerically simulated again, and the data of the scheme with the highest axial flow velocity uniformity among the 16 schemes are compared. The 16 schemes in the orthogonal experimental design are named $L_{1} \sim L_{16}$. The program parameter settings are shown in Table 4.

A representative scheme is selected for numerical simulation analysis to study the flow pattern of the water in the forebay. The accuracy of the orthogonal experiment results is analyzed and verified to further confirm the effect of the orthogonal experiment, and the selected typical schemes are shown in Table 5. 


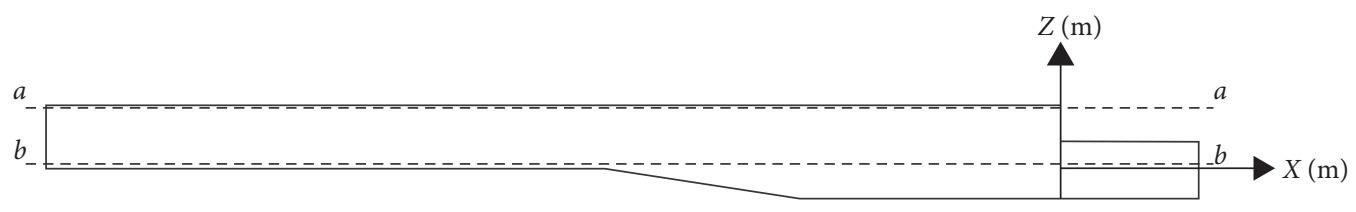

FIgURE 4: Schematic of the position of each section.

TABlE 4: Comparison of diversion pier parameter settings.

\begin{tabular}{lcccc}
\hline Schemes & & \multicolumn{2}{c}{ Diversion pier parameters } \\
& Length $l / m$ & Width $b / m$ & 10 & \\
Radian $\alpha{ }^{\rho}$ & 15 & 0.4 \\
$L_{1}$ & 7 & 0.4 & 20 & 0.6 \\
$L_{2}$ & 7 & 0.6 & 25 & 0.8 \\
$L_{3}$ & 7 & 0.8 & 15 & 1.0 \\
$L_{4}$ & 7 & 1.0 & 10 & 0.8 \\
$L_{5}$ & 8 & 0.4 & 25 & 1.0 \\
$L_{6}$ & 8 & 0.6 & 20 & 0.4 \\
$L_{7}$ & 8 & 0.8 & 20 & 0.6 \\
$L_{8}$ & 8 & 1.0 & 25 & 1.0 \\
$L_{9}$ & 9 & 0.4 & 10 & 0.8 \\
$L_{10}$ & 9 & 0.6 & 15 & 0.6 \\
$L_{11}$ & 9 & 0.8 & 25 & 0.4 \\
$L_{12}$ & 9 & 1.0 & 20 & 0.6 \\
$L_{13}$ & 10 & 0.4 & 15 & 0.4 \\
$L_{14}$ & 10 & 0.6 & 10 & 1.0 \\
$L_{15}$ & 10 & 0.8 & & 0.8 \\
$L_{16}$ & 10 & 1.0 & & \\
\hline
\end{tabular}

TABLE 5: Typical calculation scheme of the numerical model.

\begin{tabular}{lcccc}
\hline \multirow{2}{*}{ Schemes } & & \multicolumn{2}{c}{ Diversion pier parameters } \\
& Length $l / m$ & Width $b / m$ & Radian $\alpha \rho^{\circ}$ & Relative height $h$ \\
\hline$L_{4}$ & 7 & 1.0 & 25 & 1.0 \\
$L_{7}$ & 8 & 0.8 & 25 & 0.4 \\
$L_{11}$ & 9 & 0.8 & 10 & 0.6 \\
$L_{15}$ & 10 & 0.8 & 15 & 1.0 \\
\hline
\end{tabular}

4.3. Analysis of Numerical Simulation Results of Each Typical Scheme. Figure 5(a) and 5(b) show the horizontal crosssectional flow field diagrams of the surface layer and bottom layer of Scheme 4. The flow field diagram shows that the flow pattern at the bottom of the forebay is the same as that of the surface layer and the flow pattern is smooth. The diversion piers under the scheme can effectively improve the flow pattern of the side forebay. Figures 5(c) and 5(d) are the horizontal cross-sectional flow field diagrams of the surface layer and bottom layer of Scheme 7, and a low-velocity zone is formed on the left side wall of the forebay. A large-scale recirculation zone appears in the low-velocity zone of the bottom layer, and the recirculation center position is approximately at $X-6 \mathrm{~m}$. From the bottom layer to the surface layer, the recirculation center position gradually moves in the downstream direction. The range of the swirling zone gradually decreases, the surface layer forms a small-scale recirculation zone, and the recirculation center position is at $X$ of $-2 \mathrm{~m}$. The main flow line is skewed due to the large radian of the diversion pier, thereby affecting the water inlet conditions of the 1\# inlet channel. Figures 5(e) and 5(f) show the horizontal cross-section flow field diagrams of the surface layer and bottom layer of Scheme 11. The water flow velocity in the middle of the forebay is relatively large with a tendency to decrease on both sides. Moreover, a large-scale recirculation zone appears on the bottom layer. The center position of the recirculation is at $X-5 \mathrm{~m}$. From the bottom layer to the surface layer, the center of the recirculation gradually moves in the direction of the downstream flow, and the range of the swirling zone gradually decreases to form a small-scale recirculation zone on the surface layer. Comparing plan 11 with plan 7, the return center position is at $X-2 \mathrm{~m}$, and the water flow in front of the $1 \#$ inlet channel of plan 11 is smooth. Figures $5(\mathrm{~g})$ and $5(\mathrm{~h})$ show the horizontal cross-sectional flow field diagrams of the surface layer and bottom layer of Scheme 15. A large-scale recirculation zone is found in the bottom layer, and the recirculation center position is approximately at $X-6 \mathrm{~m}$. Scheme 15 has a slightly smaller bottom recirculation zone than scheme 11. From the bottom layer to the surface layer, the center position of the recirculation gradually moves in the downstream direction, and the scope of the recirculation zone gradually decreases, forming a small-scale recirculation zone on the surface layer. The center position of the surface 

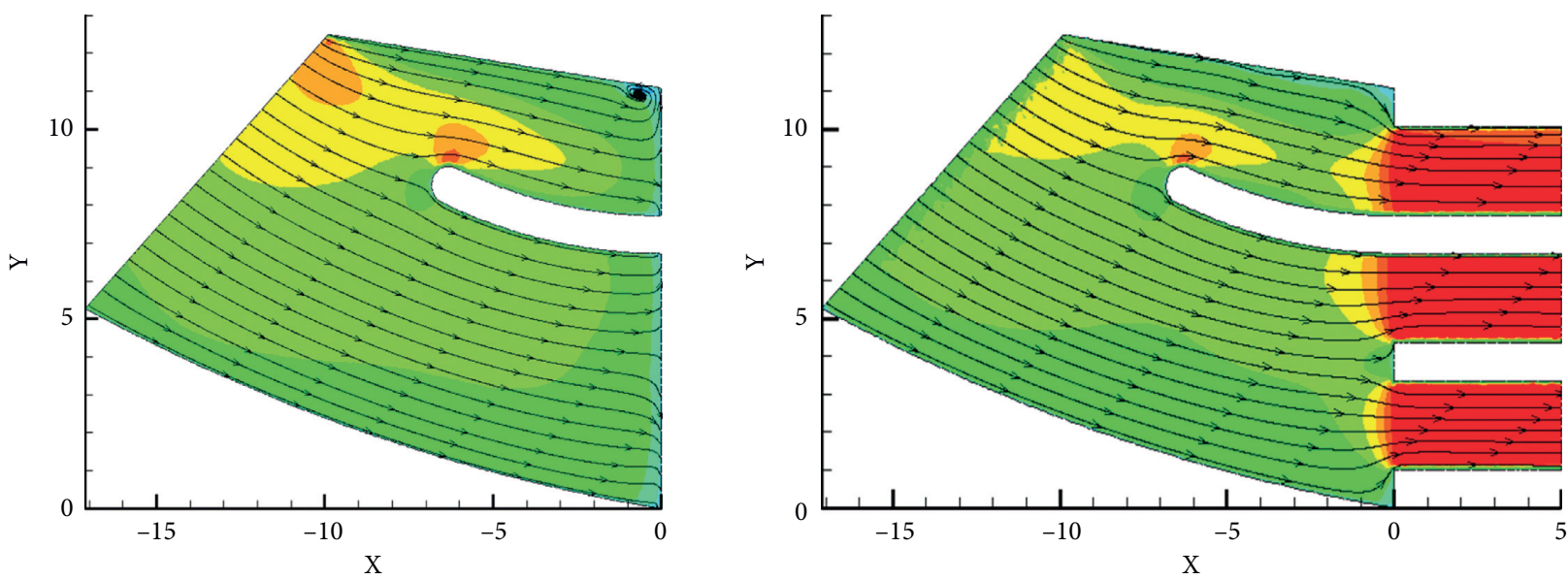

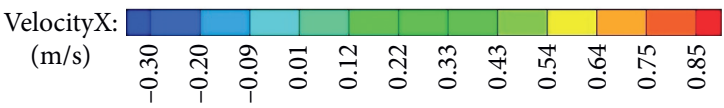

(a)
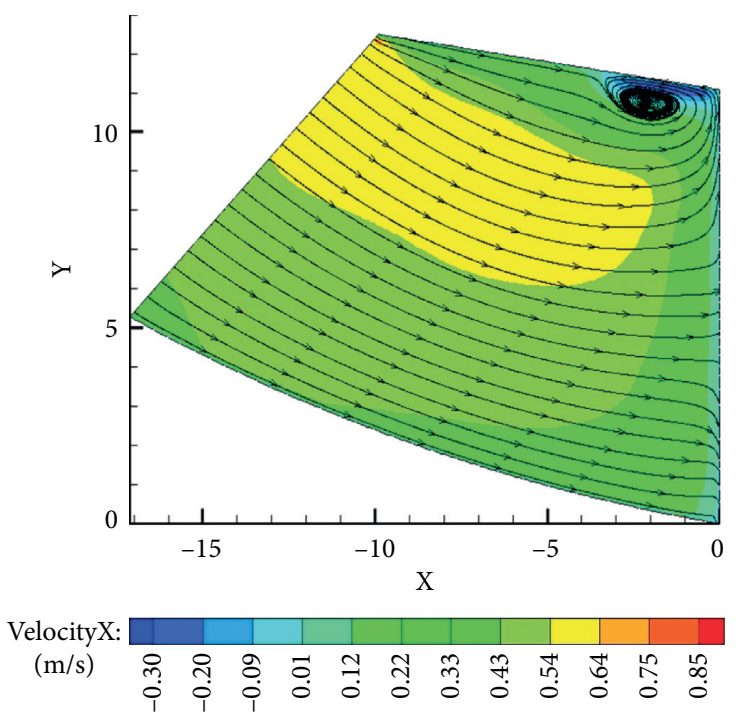

(c)

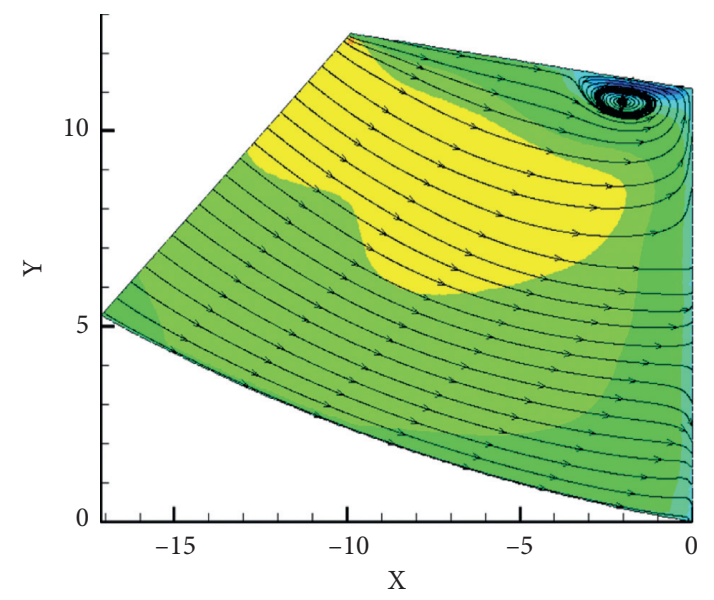

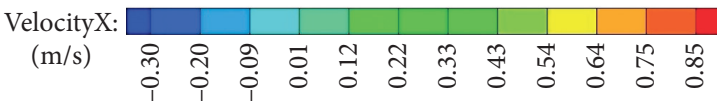

(e)

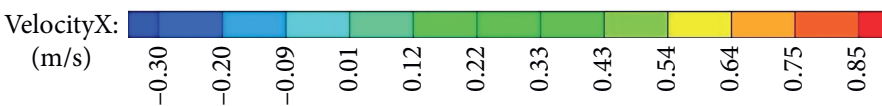

(b)

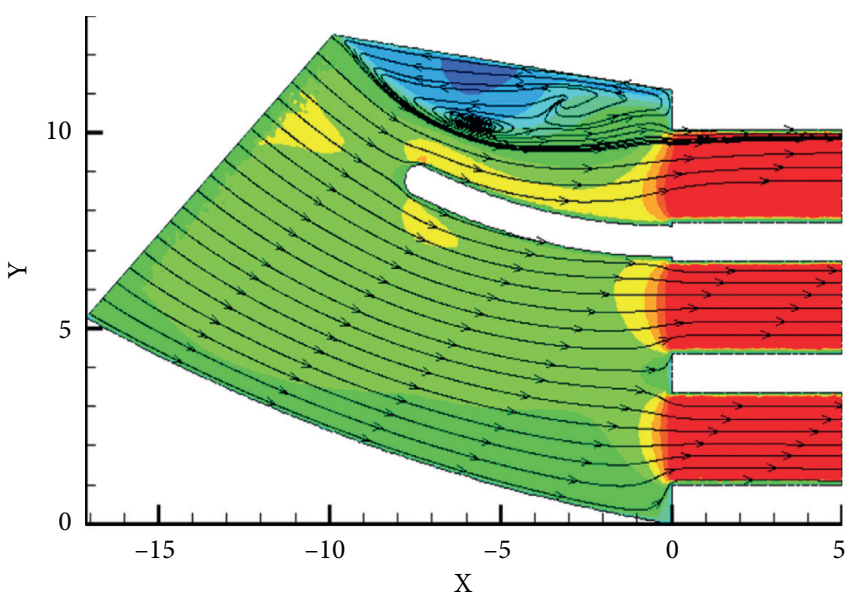

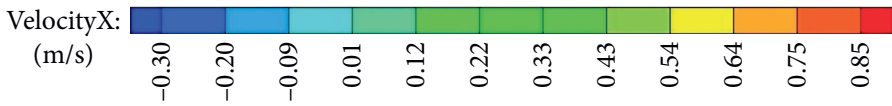

(d)

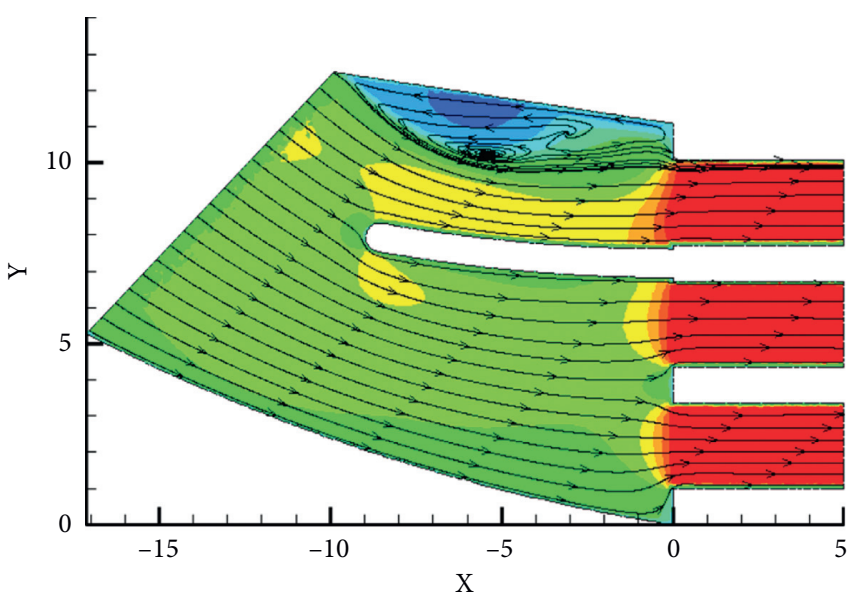

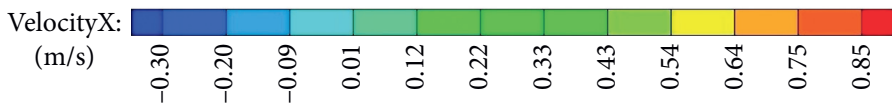

(f)

Figure 5: Continued. 

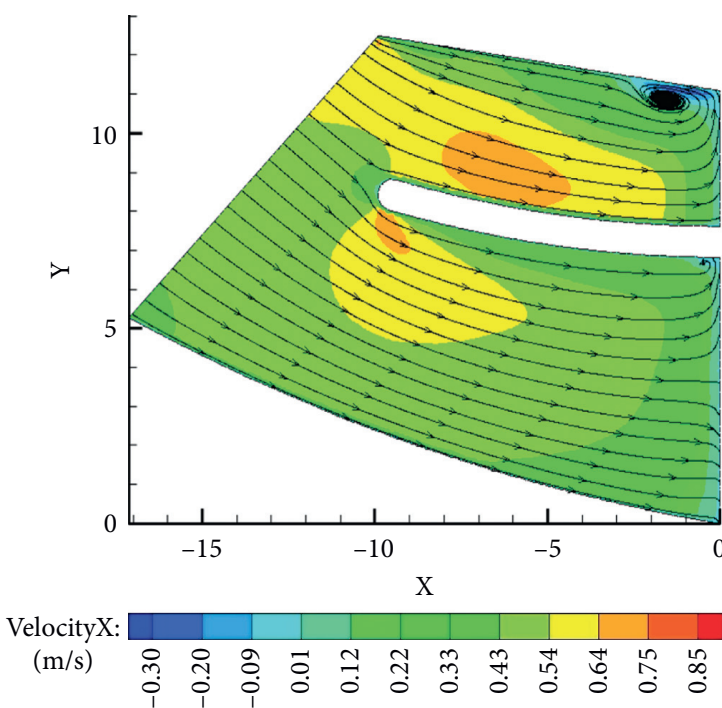

(g)

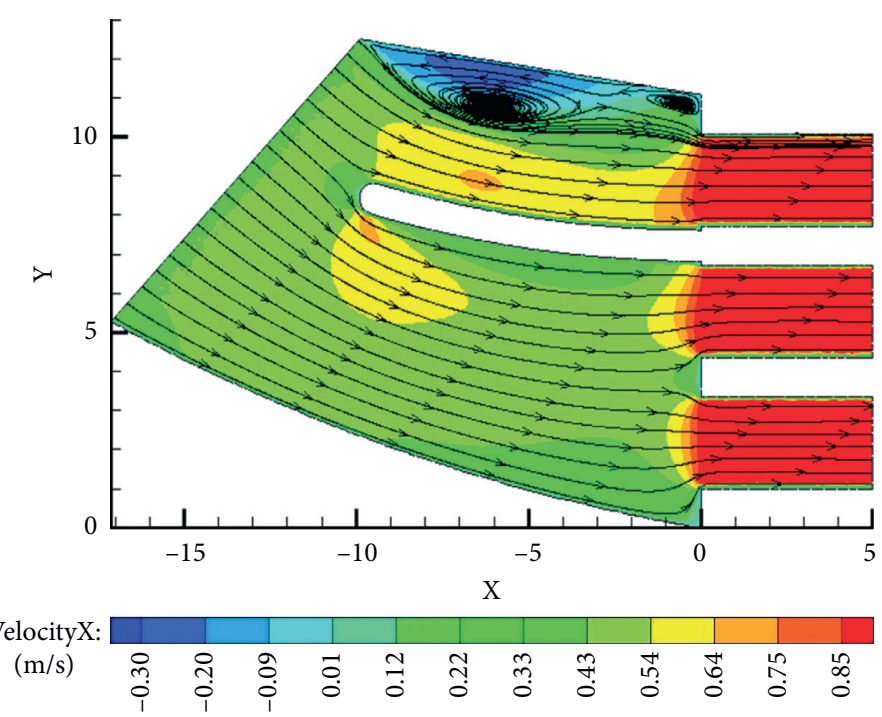

(h)

FiguRE 5: Flow field diagrams of the horizontal section of the surface layer and bottom layer of different schemes. (a) L4 surface layer flow field distribution. (b) L4 bottom layer flow field distribution. (c) L7 surface layer flow field distribution. (d) L7 bottom layer flow field distribution. (e) L11 surface layer flow field distribution. (f) L11 bottom layer flow field distribution. (g) L15 surface layer flow field distribution. (h) L15 bottom layer flow field distribution.

layer recirculation is at $-1.5 \mathrm{~m}$. The recirculation zone formed at the bottom layer is far from the inlet of the 1 \# inlet channel. Thus, it slightly affects the flow pattern of the water in front of the 1 \# inlet channel.

The axial flow field diagrams of the surface layer and bottom layer horizontal section of each typical scheme are compared and analyzed, as shown in Figure 5. In the $\mathrm{L}_{4}$ scheme of Figures 5(a) and 5(b), the surface layer and bottom layer have the smoothest flow lines and the best flow pattern, which is the optimal scheme. For the excellent scheme directly observed in the orthogonal experiment, the design scheme is observed when the index is the largest, that is, the fourth scheme $A_{1} B_{4} C_{4} D_{4}$. The maximum flow rate uniformity is $90.75 \%$, which is $7.29 \%$ higher than the original scheme. This conclusion shows that the results of orthogonal experimental design and numerical simulation analysis are consistent.

\section{Conclusion}

The rectification measures for the forebay of the pump station with a $45^{\circ}$ lateral bending angle are studied. The orthogonal test method is used to design the various geometric parameters of the diversion pier, and the optimized parameter combination is obtained by analyzing the orthogonal test results. Then, CFX numerical calculation is used to verify the analysis. Conclusions are as follows:

(1) Adding diversion piers between the 1\# and 2\# inlet channels can effectively improve the flow pattern of the forebay. Through the orthogonal experiment, the different geometric parameters of the diversion pier lead to a large difference in the uniformity of the flow velocity at the inlet section of the $1 \#$ inlet channel.
(2) Through the analysis, the range indicates that the factors from primary to secondary are DBAC. That is, among the four factors that affect the rectification effect of the diversion pier, the relative height of the diversion pier is the key factor, the length of the diversion pier and the width of the diversion pier are the general factors, and the radian of the diversion pier is the secondary factor.

(3) Various factors have different effects on the uniformity of the axial flow velocity at the inlet section of the inlet flow channel. The length of the diversion pier at $8 \mathrm{~m}$ leads to the smallest index, and the indexes below $8 \mathrm{~m}$ and above $8 \mathrm{~m}$ are large. Similarly, the diversion pier radian of $20^{\circ}$ leads to the smallest index, and the indexes below $20^{\circ}$ and higher than $20^{\circ}$ are large. As the width of the diversion pier increases, the index gradually increases, forming a trend that when the width of the diversion pier continues to increase, the index also increases. Similarly, when the relative height of the diversion pier is from 0.4 to 1.0, the index also increases, that is, when the height of the diversion pier is flushed with the water surface, the index reaches the maximum value.

\section{Data Availability}

The curve data used to support the findings of this study are available from the corresponding author upon request.

\section{Conflicts of Interest}

The authors declare that there are no conflicts of interest regarding the publication of this paper. 


\section{References}

[1] C. Liu, X. Han, J. Zhou, Y. Jin, and L. Cheng, "Numerical simulation of turbulent flow in forebay with side-intake of pumping station," Journal of Drainage and Irrigation $\mathrm{Ma}$ chinery Engineering, vol. 27, no. 05, pp. 281-286, 2009.

[2] X. Zhang, J. Zhou, J. Liang, and H. Zhang, "Experimental research on flow pattern of forebay of side-inlet pumping station," South-to-North Water Transfers and Water Science \& Technology, vol. 14, no. 3, pp. 101-105, 2016.

[3] H. Zhao, F. Yang, C. Liu, S. Chen, and J. He, "Numerical simulation of side-intake flow for fluid meliorating of pumping stations," Water Resources and Hydropower Engineering, vol. 48, no. 07, pp. 79-84, 2017.

[4] H. Xu and L. Zhang, "Review of flow pattern modifying technology for pump sump with side-inlet," Advances in Science and Technology of Water Resources, vol. 28, no. 06, pp. 84-88, 2008.

[5] C. Wang, X. Chen, N. Qiu, Y. Zhu, and W. Shi, "Numerical and experimental study on the pressure fluctuation, vibration, and noise of multistage pump with radial diffuser," Journal of the Brazilian Society of Mechanical, vol. 40, p. 481, 2018.

[6] D. Zhang, W. Jiao, L. Cheng et al., "Experimental study on the evolution process of the roof-attached vortex of the closed sump," Renewable Energy, vol. 164, pp. 1029-1038, 2021.

[7] L. Zhou, W. Wang, J. Hang, W. Shi, H. Yan, and Y. Zhu, "Numerical investigation of a high-speed electrical submersible pump with different end clearances," Water, vol. 12, no. 4 , p. 1116, 2020.

[8] L. Zhou, C. Han, L. Bai, and W. Shi, "Numerical and experimental study of multiphase transient core-annular flow patterns in a spouted bed," ASME Journal of Energy Resource Technology, vol. 142, no. 9, Article ID 092104, 2020.

[9] S. Tang, S. Yuan, and Y. Zhu, "Convolutional neural network in intelligent fault diagnosis toward rotatory machinery," IEEE Access, vol. 8, no. 1, pp. 86510-86519, 2020.

[10] S. Tang, S. Yuan, and Y. Zhu, "Deep learning-based intelligent fault diagnosis methods toward rotating machinery," IEEE Access, vol. 8, no. 1, pp. 9335-9346, 2020.

[11] S. Tang, S. Yuan, and Y. Zhu, "Data preprocessing techniques in convolutional neural network based on fault diagnosis towards rotating machinery," IEEE Access, vol. 8, pp. 149487-149496, 2020.

[12] H. Wang, Z. Qian, D. Zhang, T. Wang, and C. Wang, "Numerical study of the normal impinging water jet at different impinging height, based on wray-agarwal turbulence model," Energies, vol. 13, no. 7, p. 1744, 2020.

[13] X. He, Y. Zhang, C. Wang et al., "Influence of critical wall roughness on the performance of double-channel sewage pump," Energies, vol. 13, no. 2, p. 464, 2020.

[14] H. L. Wang, B. Long, Y. Yang, Y. Xiao, and C. Wang, "Modelling the influence of inlet angle change on the performance of submersible well pumps," International Journal of Simulation Modelling, vol. 19, no. 1, pp. 100-111, 2020.

[15] H. Wang, B. Long, C. Wang, C. Han, and L. Li, "Effects of the impeller blade with a slot structure on the centrifugal pump performance," Energies, vol. 13, no. 7, p. 1628, 2020.

[16] L. Zhou, K. Deshpande, X. Zhang, and R. K. Agarwal, "Process simulation of Chemical Looping Combustion using ASPEN Plus for a mixture of biomass and coal with various oxygen carriers," Energy, vol. 195, Article ID 116955, 2020.

[17] C. E. Sweeney, R. A. Elder, and D. Hay, "Pump sump design experience: summary," Journal of Hydraulic Division, ASCE, vol. 108, no. HY3, pp. 361-377, 1982.
[18] R. Spence and J. Amaral-Teixeira, "Investigation into pressure pulsations in a centrifugal pump using numerical methods supported by industrial tests," Computers \& Fluids \& Fluids, vol. 37 , no. 6, pp. 690-704, 2008.

[19] J. Zhou, F. Tang, C. Liu, J. Yuan, Z. Zu, and Z. Yang, "Modeling study on improving flow pattern in diffusion channel of WANGSAN pumping-station," Journal of Drainage and Irrigation Machinery Engineering, vol. 12, no. 2, 1995.

[20] L. Cheng, W. Qi, C. Luo, Y. Shang, and H. Yuan, "Effect of geometric parameters of Y-shaped diversion piers on flow pattern in forebay of pumping station," Advances in Science and Technology of Water Resources, vol. 34, no. 01, pp. 68-72, 2014.

[21] L. Zhou, W. Shi, W. Lu, R. Xu, and C. Wang, "Orthogonal test and optimization design of submersible pump guide vanes," Journal of Drainage and Irrigation Machinery Engineering, vol. 29, no. 04, pp. 312-315+342, 2011.

[22] L. Shi, F. Tang, X. Liu, R. Xie, X. Song, and W. Zhang, "Optimization design and experiment of large cube-type pump device," Transactions of the Chinese Society for Agricultural Machinery, vol. 48, no. 01, pp. 96-103, 2017.

[23] L. Shi, J. Zhu, F. Tang, and C. Wang, "Multi-disciplinary optimization design of axial-flow pump impellers based on the approximation model," Energies, vol. 13, no. 4, p. 779, 2020.

[24] L. Shi, W. Zhang, H. Jiao et al., "Numerical simulation and experimental study on the comparison of the hydraulic characteristics of an axial-flow pump and a full tubular pump," Renewable Energy, vol. 153, pp. 1455-1464, 2020. 